\title{
Polivictimización reportada por escolares chilenos: ¿es la escuela un lugar donde sentirse a salvo?
}

\section{Poly-victimization reported by Chilean schoolchildren: is school a place to feel safe?}

\section{Polivitimização relatada por escolares chilenos: a escola é um lugar} para se sentir seguro?

\author{
Carlos Rodríguez Garcés \\ Universidad del Bío-Bío \\ Chillan, Chile \\ carlosro@ubiobio.cl \\ (D) ORCID: https://orcid.org/0000-0002-9346-0780. \\ Claudia Suazo Ruíz \\ Universidad del Bío-Bío \\ Chillan, Chile \\ claudiasuazo1601@ubiobio.cl \\ D ORCID: https://orcid.org/0000-0002-9937-4875 \\ Geraldo Padilla Fuentes \\ Universidad del Bío-Bío \\ Chillan, Chile \\ gpadilla@ubiobio.cl \\ (D) ORCID: https://orcid.org/0000-0003-0882-1818 \\ Denisse Espinosa Valenzuela \\ Universidad del Bío-Bío \\ Chillan, Chilee \\ daespinosa@ubiobio.cl \\ (D) ORCID: https://orcid.org/0000-0003-1486-7046
}

Recibido - Received - Recebido: 15 / 09 / 2021 Corregido - Revised - Revisado: 09 / 11 / 2021 Aceptado - Accepted - Aprovado: 25 / 11 / 2021

DOI: https://doi.org/10.22458/ie.v24i36.3918

URL: https://revistas.uned.ac.cr/index.php/innovaciones/article/view/3918

\begin{abstract}
Resumen: La exposición a episodios de violencia, ya sea en el barrio, la familia o la escuela, tiende a formar parte de la cotidianidad de niños, niñas y adolescentes (NNA). En tal sentido, el objetivo de este artículo es analizar la simultaneidad y frecuencia de los eventos de violencia reportados por los NNA en calidad de víctimas, fenómeno tipificado como polivictimización. A nivel muestral, hace uso de la Primera Encuesta Nacional de Polivictimización aplicada a estudiantes chilenos/as entre $7^{\circ}$ Básico y $3^{\circ}$ Medio, aplicando un diseño metodológico de análisis estadístico inferencial y de regresión logística, estimando el nivel de vulnerabilidad y violencia a la que están expuestos los NNA y sus factores de protección y riesgo. Los resultados muestran que la victimización y polivictimización es declarada por gran parte de los y las participantes, lo que haría de ambas problemáticas algo transversal en el sistema educativo. Sobresalen los delitos comunes, la victimización indirecta y la realizada por pares, especialmente la recepción o atestiguamiento de ataques, golpes y amenazas. Las conclusiones destacan la necesidad de medidas integrales que tengan a la escuela como espacio crucial de fomento a la sana convivencia y rechazo de la violencia en cualquiera de sus formas.
\end{abstract}

Palabras clave: Abuso de menores, coexistencia pacífica, polivictimización, estudiantes, gestión educacional, educación.

\begin{abstract}
Exposure to episodes of violence, whether in the neighborhood, family or school, tends to be part of the daily lives of children and teenagers (NNA). In this sense, the objective of this article is to analyze the simultaneity and frequency of violent events reported by children and teenagers as victims, a phenomenon typified as poly-victimization. At the sample level, it makes use of the First National Polivictimization Survey
\end{abstract}


applied to Chilean students between 7th grade and 3rd grade, applying a methodological design of an inferential statistical analysis and logistic regression, estimating the level of vulnerability and violence to which children and teenagers are exposed to and their protection and risk factors. Results show that participants claimed to have experienced victimization and poly-victimization, turning both problems into something transversal in the educational system. Common crimes and indirect victimization carried out by peers stand out, especially in witnessing attacks, hittings, and threats. Conclusions highlight the need for comprehensive school measures as a crucial space for promoting healthy coexistence and violence rejection in any of its forms.

Keywords: child abuse, peaceful coexistence, poly-victimization, students, educational management, education.

Resumo: A exposição a episódios de violência, seja no bairro, na família ou na escola, tende a fazer parte do dia a dia de crianças e adolescentes (CA). Nesse sentido, o objetivo deste artigo é analisar a concomitância e a frequência dos eventos de violência relatados por crianças e adolescentes como vítimas, fenômeno tipificado como polivitimização. No nível de amostra, faz-se uso da Primeira Pesquisa Nacional de Vitimização, aplicada a alunos chilenos da $7^{\text {a }}$ série à $12^{\mathrm{a}}$ série, aplicando um desenho metodológico de análise estatística inferencial e regressão logística, estimando o nível de vulnerabilidade e violência à qual as crianças e adolescentes estão expostos e seus fatores de proteção e risco. Os resultados revelam que a vitimização e a polivitimização foram declaradas por grande parte dos participantes, o que tornaria ambos os problemas transversais ao sistema educacional. Destacam-se os crimes comuns, a vitimização indireta e perpetrada por pares, com destaque para receber ou testemunhar agressões, pancadas e ameaças. As conclusões evidenciam a necessidade de medidas integrais onde a escola seja um espaço fundamental para a promoção da convivência saudável e o repúdio à violência em qualquer forma.

Palavras-chave: Maus-tratos infantis, convivência pacífica, polivitimização, estudantes, gerenciamento educacional, educação.

\section{INTRODUCCIÓN}

El bienestar físico y psicológico de niños, niñas y adolescentes (NNA) se ha transformado en pilar de las políticas educativas en Chile. Las medidas legislativas y programáticas de la última década han estado especialmente orientadas al diagnóstico y tratamiento de hechos de violencia, maltrato y/o discriminación en la escuela, en el entendido de que los procesos de enseñanza-aprendizaje deben estar acompañados por climas de convivencia seguros y organizados. Si bien estas iniciativas surgen y se desarrollan en el contexto nacional, fueron influenciadas por la alerta internacional en torno a la importancia de erradicar la violencia de las escuelas (Echeverri y Vargas-González, 2018).

Si bien la victimización y la violencia son fenómenos que adquieren connotaciones particulares dependiendo de las características de la población en donde se manifiestan, hay aspectos generales que permiten su estudio e intervención. Factores culturales y diversos elementos de los contextos en que viven las personas pueden potenciar o mitigar la presencia de estas problemáticas, como son los niveles de pobreza socioeconómica y la accesibilidad a servicios sociales. Si pusiéramos atención, podríamos encontrar violencia diariamente en cualquier sitio, en las actividades que realizamos y a nuestro alrededor, incluso en los medios de comunicación, los videojuegos o las redes sociales, aunque ello no puede invisibilizar el hecho de que sus manifestaciones más crudas afectan a los sectores y grupos más vulnerables. Por lo demás, dependiendo del uso que hagamos o veamos en la violencia, es que decidimos actuar o no contra ella, puesto que, en ocasiones, su práctica puede justificarse en nombre del bien común o la protección de otros (Cañas-Pardo, 2017).

Todo esto hace de los hechos de violencia y victimización poliedros multidimensionales que, más allá de ser pura manifestación, se nutren de diversos flancos y obedecen a factores interrelacionados a nivel personal, relacional y de contextos. Cuando incrementa la violencia en algunos sectores, la escuela no puede evitar ser un escenario más de vulneración al recibir la influencia de lo que suceden en el barrio o la familia. Sin embargo, en su objetivo por educar en contenidos y en valores, la escuela también tiene la oportunidad de constituirse en un lugar de protección y resguardo para los NNA, aunque corre el riesgo que sus planes de convivencia pacífica no sean efectivos debido a la violencia circundante. 
El maltrato escolar, la violencia y la victimización son más propensos de ocurrir en climas de convivencia rodeados por la vulnerabilidad y/o naturalización de estas prácticas. Si expandimos un poco la mirada, la escuela es uno más de los sitios donde pasan el tiempo y comparten relaciones los NNA, por lo que una escuela con climas áulicos o de convivencia general conflictivos puede ser el reflejo de situaciones de vulnerabilidad arraigadas en el barrio, en la casa o en la socialización que se entabla con amigos/as. Además, estas experiencias lastran su capacidad para constituirse en un refugio donde los y las estudiantes puedan sentirse emocionalmente a salvo.

Los problemas de acoso, ataques o abuso entre pares constituyen actualmente una preocupación dentro del contexto escolar chileno, similar a lo que sucede en varios países del mundo. El estudio de la violencia en los distintos establecimientos educativos del país se ha vuelto un insumo clave para la toma de decisiones, además de que algunos episodios de violencia escolar se han vuelto mediáticos, dejando en claro la necesidad por conocer quiénes están involucrados y cuál sería el origen o motivación de estos actos (Salgado, 2017). Por lo demás, estas acciones no tan solo ponen en riesgo el bienestar individual físico y psicológico de los NNA, sino que afectan al ecosistema donde estos comparten y aprenden cómo relacionarse con otros, más al considerar que la victimización escolar y el maltrato entre pares se presenta simultáneamente en distintos lugares del país, sin diferenciar entre sectores sociales o clases (Garaigordobil y Oñederra, 2008).

Usualmente, los episodios de victimización aparecen en ambientes comunes al interior de los establecimientos, como son el patio de recreo, los baños, pasillos o el comedor, donde la supervisión por parte del profesor o inspectores es menor debido a la cantidad de alumnos (Tresgallo, 2011; Salgado, 2017). Eso sí, no podemos obviar que las situaciones de maltrato entre pares no siempre se dan dentro de los contextos educativos, pudiendo producirse en otros ambientes que la víctima frecuenta, como el barrio, alguna plaza o en la calle. Sin embargo, la escuela es un sitio crucial para trabajar en la prevención y gestión de la violencia en sus múltiples formas, dado que junto con reunir a las distintas familias puede educar a los NNA con herramientas pacíficas para la resolución de conflictos. El complemento que puede realizar a los esfuerzos familiares por proteger a los NNA es lo que potencialmente hará la diferencia entre naturalizar las prácticas de agresión o acoso y el rechazar sus manifestaciones a favor de la conversación y el diálogo.

No podemos dejar fuera de todo esto la influencia que variables afectivas y emocionales tienen sobre el ejercicio y apreciación de la victimización. Una de las características que comparten tanto agresores como víctimas durante la pubertad y adolescencia es la inestabilidad emocional, la falta de habilidades sociales y el intento por forjar una identidad propia, componentes que pueden generar conductas agresivas y de impulsividad frente a situaciones de estrés o amenaza. Esto hace variar el modo en que se comprende la violencia que circunda a los NNA, ya que, dependiendo del estado afectivo o anímico, sus reacciones podrían ser exageradas o desensibilizadas frente a cualquier estímulo. Tal y como sucede con algunos NNA que muestran una actitud pasiva frente a la recepción o atestiguamiento de violencia, ya sea por costumbre o resignación, hay otros que responden activamente a cualquier intento o señal de amedrentamiento. Eso sí, cabe destacar que, usualmente, quienes más sufren de victimización en la escuela son los y las estudiantes que demuestran un bajo nivel de autoestima, se comportan introvertidamente y no presentan gran resistencia a los abusos (Tur-Porcar, Mestre, Samper y Malonda, 2012).

A lo anterior se añade la importancia que tiene el modo de actuar de quienes observan en la aceptación y repetición de violencia. La victimización en la escuela es un fenómeno grupal e interpersonal, donde el resto de compañeros/as o amigos/as puede actuar de modo pasivo, como defensores de las víctimas, instigadores de los hechos o quienes de inmediato buscan ayuda en algún adulto cercano (Cañas-Pardo, 2017). Por supuesto, el rol de los profesores/as y profesionales asistentes de la educación es clave, ya que son ellos/as quienes, desde el interior del aula y el colegio, pueden controlar que los insultos, agresiones psicológicas y/o golpes se detengan, siempre y cuando vean en estos hechos algo repudiable y no una 
simple mímesis de lo que ven en televisión o en sus barrios (Rojas y Sierra, 2013; Villalobos y otros, 2016; Castañeda, 2018).

En razón de lo expuesto, el principal interés de esta investigación se sitúa en el análisis de la victimización y violencia en la escuela desde una perspectiva multidimensional. En tal sentido, las aportaciones de Finkelhor, Hamby, Ormrod y Turner (2005) son esenciales, puesto que a través del Cuestionario Juvenil de Victimización (JVQ) plantean la medición de la polivictimización.

El fenómeno de la violencia en NNA tiende a ser abordado desde múltiples acercamientos teóricos y metodológicos, elaborándose diferentes constructos para comprenderlo y analizarlo. Dentro de los más relevantes y clásicos encontramos el "abuso infantil" que refiere a la situación donde se agrede intencionalmente a un menor de edad, ya sea de forma física, psicológica o sexual (Losada y Porto, 2019). Por otra parte, hallamos el concepto de "revictimización" o "victimización secundaria" que hace mención a la exposición de la víctima a revivir la situación traumática en un proceso de investigación policial y/o judicial desprolijo (Subijana y Echeburúa, 2018). Posteriormente, los acercamientos teóricos más actuales han desarrollado el constructo de "polivictimización", es decir, la exposición recurrente, múltiple y diversa que los NNA tendrían hacia la violencia en sus distintos formatos de manera simultánea y en contextos como la escuela, el barrio o el hogar que podrían generar un trauma acumulativo (Guerra, Inostroza, Villegas, Villalobos y Pinto-Cortez, 2017; Finkelhor, Hamby, Ormrod, \& Turner, 2005). La polivictimización comprende que victimización y violencia pueden ser condiciones de vida para algunos/as, lo que no solo apareja perjuicios corporales a causa de golpes o ataques, sino también vulneraciones indirectas, verbales, afectivas y/o sexuales, que calan profundo en la valoración que los NNA hacen de sí mismos (Pereda y Forns, 2007; Chan, Yan y Brownridge, 2013).

Debido a la gravedad que implica sufrir polivictimización, por cuanto acarrea problemas de salud mental, afectiva, emocional y cognitiva entre NNA (Finkelhor, Shattuck, Turner, Ormrod y Hamby, 2011), el objetivo de este artículo es describir y analizar la situación de polivictimización que reportan estudiantes chilenos/as en la Primera Encuesta Nacional de Polivictimización (EPV) entre niños, niñas y adolescentes (NNA), adaptación del Cuestionario Juvenil de Victimización (JVQ) de los investigadores Finkelhor, Hamby, Ormrod y Turner (2005). A través de estadística inferencial con contraste de asociación y análisis multivariante, específicamente con la confección de dos modelos de regresión logística binomial, se indagan las diferentes dimensiones e indicadores que componen la EPV. En lo específico, los propósitos de este estudio se orientan a caracterizar el fenómeno de la polivictimización, perfilar las tipologías más recurrentes y sus atributos distintivos y, por último, exponer los factores de riesgo y/o protección que incrementan o disminuyen la manifestación de la polivictimización en NNA.

\section{MATERIALES Y MÉTODOS}

Con el objetivo de reportar -en términos descriptivos e inferenciales- la situación de victimización entre NNA, este artículo examina, desde una perspectiva cuantitativa, las distintas dimensiones que componen la EPV realizada en 2017 entre la población estudiantil. Mediante una serie de indicadores estadísticos, se cuantifican los niveles de exposición que presentan los NNA ante los distintos eventos de victimización en términos de prevalencia, recurrencia e incidencia, buscando reconocer eventuales factores de riesgo y/o de protección frente a estas situaciones de vulnerabilidad en la escuela, en el barrio o en el hogar.

La Encuesta Nacional de Polivictimización (EPV) corresponde a una adaptación al castellano "Juvenile Victimization Questionnaire" (JVQ) desarrollado originalmente por los investigadores Finkelhor, Hamby, Ormrod y Turner (2005). Ha sido sometida a múltiples procesos de validación, tanto en su versión de inglés original como en su traducción y adaptación al castellano, mostrando adecuados índice de ajuste 
y de validez interna. A este respecto, destacan particularmente los estudios de validación de Kretschmar Tossone, Butcher y Flannery (2017) y Sterzing, Ratliff, Gartner, McGeough y Johnson (2017), quienes explorando una versión recortada de la misma escala, encuentran adecuados índices de ajuste. Por otra parte, se halla la validación de la versión china realizada con estudiantes de secundaria, efectuada por Cheng, Cao, Liu y Chen (2010), que obtuvo un Alfa de Cronbach de 0,75 y una fiabilidad test-retest de 0,82 , lo que demuestra que el instrumento es válido y confiable para ser aplicado a estudiantes de secundaria en China.

En su versión en castellano, destacan los procesos de validación realizados por Kirchner, Soler y Paretilla (2013), quienes, mediante un análisis factorial confirmatorio y de consistencia interna a una muestra de 553 adolescentes, obtienen un Alfa de Cronbach de 0,82 y 0,84, según sea referido al último año y/o a lo largo de la vida, concluyendo que el JVQ es un instrumento útil para funciones de cribado, especialmente para las victimizaciones ocurridas en el último año. Dentro de los estudios de validación más recientes, se pueden mencionar el realizado por el Grupo de Investigación en Victimización Infantil y Adolescente de la Universidad de Barcelona, específicamente por los investigadores Pereda, Gallardo y Guilera (2018), quienes igualmente reconocen las propiedades métricas del instrumento. Para el caso chileno, destacan los estudios realizados por Pinto, Flores, Pereda y Guerra (2019).

La EPV fue aplicada a 19.867 estudiantes entre $7^{\circ}$ Básico y $3^{\circ}$ Medio, distribuidos entre 699 unidades educativas de distinta dependencia administrativa y localización en el país. La definición de esta población objetivo se hace sobre la base de consideraciones teóricas y metodológicas que refieren a la relativa homogeneidad pretendida en la muestra a fin de garantizar una estandarización y equivalencia en los niveles de comprensión de los reactivos del instrumento. Por tal motivo, se excluyen estudiantes de menor edad por las eventuales dificultades que presentaría la comprensión del instrumento y abordaje de la problemática para este grupo. Por otra parte, también se excluye a los estudiantes de $4^{\circ}$ medio, por cuanto estos se encuentran en la fase terminal de su proceso educativo y abocados a preparar la prueba de admisión universitaria.

El levantamiento de información de la EPV fue realizado mediante un cuestionario autoaplicado con asistencia de facilitador durante los meses de octubre a diciembre 2017 a una muestra probabilística estratificada en tres etapas. La primera unidad de estratificación fueron los establecimientos educacionales de todo el país, seleccionándose un total de 699 de ellos; en un segundo nivel, se estratificó por curso (de $7^{\circ}$ básico a $3^{\circ}$ medio), para, finalmente, seleccionar a estudiantes de cada curso.

El índice de respuesta a la EPV es del orden del 99,1\% y considera variables sociodemográficas, de las unidades educativas y sobre victimización. Presenta un error muestral de \pm 0.7 puntos porcentuales con varianza máxima y $95 \%$ de confianza. La EPV considera, además, una ponderación de la muestra con parámetros de ajuste post-estratificación por sexo, edad, dependencia administrativa del establecimiento y región.

En cuanto a las características de la muestra (Tabla 1), encontramos una homogeneidad en términos de género y un leve predominio de estudiantes de enseñanza media (55\%) y adolescentes entre 14 y 15 años (38,5\%). En cuanto a los niveles de representación étnica y de estudiantes con Necesidades Educativas Especiales, alcanzan el 15,5\% y 12,6\%, respectivamente. 
TABLA 1

Descripción de la muestra

\begin{tabular}{|c|c|c|c|}
\hline & $\%$ & & $\%$ \\
\hline Sexo & & Nivel educativo & \\
\hline - Hombre & 49,4 & - Secundaria & 55 \\
\hline - Mujer & 50,6 & - Primaria & 45,0 \\
\hline Edad & & IVE & \\
\hline - 16 años o más & 33,1 & - Bajo & 33,8 \\
\hline - 14 y 15 años & 38,5 & - Medio & 33,3 \\
\hline - 12 y 13 años & 28,5 & - Alto & 32,8 \\
\hline Presenta NEE & & Pertenencia étnica & \\
\hline - Sí & 12,6 & - Sí & 15,5 \\
\hline - No & 87,4 & - No & 84,5 \\
\hline Escala Rosenberg & & & \\
\hline - Adecuada & 13,8 & $\begin{array}{l}\text { Escala Birleson } \\
\text { _ Moderada o alta }\end{array}$ & 297 \\
\hline - Moderada & 74,9 & - Moderada o alta & $\begin{array}{l}29,1 \\
70,3\end{array}$ \\
\hline - Baja & 11,3 & - Ninguna o baja & \\
\hline
\end{tabular}

Este trabajo utiliza datos pertenecientes a la Primera Encuesta Nacional de Polivictimización en Niños, Niñas y Adolescentes (EPV), realizada en Chile a finales de 2017, la cual estuvo a cargo de la Dirección de Estudios Sociales (DESUC) y el Programa de Estudios Sociales del Delito, del Instituto de Sociología de la Pontificia Universidad Católica de Chile.

La encuesta se compone de 32 victimizaciones o indicadores, organizados en 6 dimensiones: Delitos comunes (A), Efectuadas por Cuidadores (B), Efectuadas por Pares (C), Sexuales (D), Indirectas (E) y Digitales (F). La dimensión de Delitos comunes se divide según exista uso de fuerza física (A1) o no (A2), mientras que la victimización Indirecta reconoce entre exposición a violencia en la comunidad (E1) o la familia (E2).

El objetivo de la EPV es determinar la magnitud de la exposición a violencias entre NNA a lo largo del país, distinguiendo según tipologías de victimización y perfiles de polivictimización. Este instrumento es una adaptación del Juvenile Victimization Questionnaire (JVQ) desarrollado por Finkelhor, Hamby, Ormrod y Turner (2005), el cual plantea una perspectiva comprensiva y multidimensional para la investigación del maltrato y experiencias de victimización durante la infancia y adolescencia. En términos generales, es una herramienta para recabar información de un amplio rango de victimizaciones, entregando una cuantificación de las principales vulneraciones que han vivido los NNA alguna vez en su vida o durante el último año.

La recolección de datos se realizó en formato autoaplicado con asistencia de facilitador. El muestreo fue de tipo probabilístico estratificado, trietápico según establecimiento > cursos > estudiantes. El error muestral es de $\pm 0,7$ puntos porcentuales, con varianza máxima y $95 \%$ de confianza.

El análisis de la EPV comienza por un abordaje panorámico a sus dimensiones e indicadores, describiendo las cifras de victimización vivida por NNA durante el último año. Como variable de comparación, se utilizaron los perfiles de polivíctima según percentil de distribución (P92) y acumulación de indicadores (Umbrales).

Uno de los objetivos sustanciales de la encuesta es identificar a los NNA polivíctimas, es decir, a aquellos jóvenes que están expuestos a múltiples hechos de vulneración en la escuela, el barrio o el hogar. Hay dos formas de establecer la condición de polivíctima. La primera, y más utilizada entre quienes analizan 
la EPV, es tomar al 8\% superior de la distribución de victimizaciones durante el último año, estableciendo como punto de corte para la polivictimización el número de indicadores reportados hasta ese percentil 92. La otra forma es considerar polivíctimas a quienes están sobre el umbral de 3 victimizaciones, distinguiendo además la intensidad de su condición, sea mediana (4-5 indicadores) o alta (más de 5 indicadores).

Ambos perfilamientos de polivictimización son utilizados a lo largo de esta investigación para contrastar y precisar algunos resultados. De igual modo, no se trabajó con la totalidad de indicadores que componen la EPV, sino que se consideraron tan solo aquellos ítems que pudiesen ocurrir en los espacios educativos. Por esto es que las cifras generales de cada Dimensión o Indicador se acompañan con su debida ocurrencia en el colegio, espacio que se distingue del barrio y la familia en múltiples aspectos. De esta forma, de los 32 indicadores originales, se utilizaron 24, lo que significó recalcular las dimensiones del instrumento siguiendo las mismas directrices de la confección original, además de excluir la victimización digital.

El interés de investigación estuvo centrado en los perfiles de polivictimización, exponiendo datos descriptivos y causales de naturaleza inferencial. Primero, se caracterizan los perfiles de polivictimización sobre la base de los criterios del rango percentil (P92) y nivel de umbrales según variables como género, la edad, el nivel de vulnerabilidad de los establecimientos, la dependencia administrativa, niveles de autoestima y depresión, contraste estadístico basado en chi cuadrado que contempla variables que según literatura especializada son relevantes. En la segunda parte, y a fin de aportar mayor profundidad en el comportamiento antes descrito, se pone a prueba la capacidad explicativa de dos modelos de regresión logística binomial, según perfiles de polivictimización.

\section{DISCUSIÓN DE RESULTADOS}

La victimización y polivictimización se configura como una problemática transversal al sistema educativo cuya influencia irradia desde y hacia la familia y el barrio. Particularmente relevantes son las victimizaciones por delitos comunes y las manifestaciones de violencia realizadas por pares, tales como ataques, golpes y amenazas.

Asumiendo que la polivictimización es un constructo complejo, la Tabla 2 proporciona dos criterios para su medición: rango percentil (P92) y umbrales. Ambos perfiles fueron construidos sobre la base de aquellas victimizaciones que pudieran haber ocurrido en la escuela durante los últimos doce meses (véase Procedimiento). Según el criterio de percentiles (P92), las polivíctimas se encontrarían principalmente expuestas a victimización por delitos comunes (98,3\%), eventos indirectos (97,9\%), pares (86,0\%), cuidadores $(82,0 \%)$ y amenazas $(74,5 \%)$, hechos que implican riesgo tanto corporal como psicológico. Estas dimensiones manifiestan prevalencias mayoritariamente sobre el 70\%, con excepción de cuidadores $(8,5 \%)$, cuyos indicadores están dirigidos principalmente al contexto familiar. 
TABLA 2

Descripción (\%) de la polivictimización durante el último año, según rango percentil y umbrales por dimensiones

\begin{tabular}{|c|c|c|c|c|c|c|}
\hline \multirow{3}{*}{$\begin{array}{c}\text { Polivictimización por } \\
\text { Dimensiones }\end{array}$} & \multirow{2}{*}{\multicolumn{2}{|c|}{$\begin{array}{l}\text { Rango percentil } \\
\text { (P92) }\end{array}$}} & \multicolumn{4}{|c|}{ Umbrales ${ }^{b}$} \\
\hline & & & \multicolumn{2}{|c|}{ Mediana } & \multicolumn{2}{|c|}{ Alta } \\
\hline & $\%$ & Escuela $^{a}$ & $\%$ & Escuela & $\%$ & Escuela \\
\hline - Delitos comunes & 98,3 & 70,5 & 76,6 & 72,3 & 97,2 & 70,2 \\
\hline - Amenazas & 74,1 & 76,2 & 30,4 & 79,9 & 67,6 & 77,9 \\
\hline - Cuidadores & 82 & 8,5 & 52 & 6,5 & 77,8 & 8,4 \\
\hline - Pares & 86 & 90,3 & 43,9 & 93,2 & 79,9 & 91,1 \\
\hline - Sexuales & 27,1 & 30,8 & 7,6 & 31,6 & 22,2 & 31,1 \\
\hline - Indirecta & 97,9 & 52,2 & 90,1 & 46,2 & 96,7 & 51,8 \\
\hline - Exposición VIF & 31,9 & 1 & 10,3 & - & 27,1 & 0,8 \\
\hline
\end{tabular}

Nota: ${ }^{a}=$ La información corresponde al indicador de la dimensión con más alta recurrencia en la escuela; ${ }^{b}=$ La definición de polivíctima se divide según acumulación de indicadores, distinguiendo entre Mediana intensidad (4-5 indicadores) y Alta intensidad (6 o más indicadores); VIF= Violencia Intrafamiliar.

Fuente: Primera Encuesta Nacional de Polivictimización, 2017.

El comportamiento de la polivictimización, según umbrales, varía en razón de la categoría de referencia. Si nos fijamos en quienes declararon entre 4 y 5 victimizaciones, es decir, polivíctimas de mediana intensidad, las cifras reflejan una vulnerabilidad más focalizada y moderada que la descrita anteriormente. Junto con disminuir los niveles de victimización, esta categoría se vincula con acciones indirectas (90,1\%) y delitos comunes (76,6\%). Sobre la base del indicador, la escuela decayó ligeramente como espacio de riesgo, albergando principalmente situaciones de violencia por pares $(93,2 \%)$, amenazas $(79,9 \%)$ y delitos comunes $(72,3 \%)$.

Sobre el perfil de polivíctima de alta intensidad, es decir, quienes reportan más de 5 indicadores, las cifras no se distanciaron significativamente de lo encontrado con P92. Se repitió la jerarquía con que fueron declaradas las primeras cinco dimensiones, disminuyendo relativamente la victimización por pares $(79,9 \%)$, cuidadores $(77,8 \%)$ y amenazas $(67,6 \%)$. En relación con la escuela, tampoco se observaron diferencias relevantes con lo dicho para P92.

Debido a esta similitud entre P92 y polivictimización de alta intensidad, la Tabla 3 considera solamente al primer perfil en la indagación de aquellos indicadores o situaciones que representarían la cotidianidad para algunos NNA, especialmente en la escuela. En términos específicos, y siguiendo las cifras de mayor prevalencia, la polivictimización sería el reflejo de vivencias como ataque físico sin uso de armas (83,8\%), atestiguamiento de balaceras, microtráfico, peleas entre pandillas (82,7\%) o discriminación por alguna característica propia (82,3\%), recibir insultos por parte de algún adulto cercano $(74,5 \%)$ y encontrarse expuesto a amenazas de ataque físico $(60,6 \%)$, situaciones que implican violencia física, verbal y simbólica en magnitudes preocupantes.

Respecto a la escuela como escenario de victimización, se observó que, pese a no ser los indicadores más frecuentes a nivel general, existen algunos eventos de la EPV que podrían llegar a estimarse consustanciales al clima de convivencia escolar de los NNA polivíctimas, como son los insultos (90,3\%), el destrozo de objetos personales $(70,7 \%)$ y recibir ataques físicos $(68,3 \%)$, los cuales son parte nuclear del maltrato escolar. Esto ubica a los compañeros y/o compañeras de escuela en una posición clave para la comprensión de la polivictimización, puesto que tendrían un rol activo en el ejercicio de la violencia (física, verbal o simbólica), pudiendo actuar también como encubridores de ella cuando son testigos. 
TABLA 3

Indicadores más reportados durante el último año por NNA polivíctimas según P92 y dimensión (\%)

\begin{tabular}{lcc}
\multicolumn{1}{c}{ Indicadores por dimensión } & $\%$ & $\begin{array}{c}\text { Ocurrencia en la } \\
\text { escuela }\end{array}$ \\
Delitos comunes & 83,8 & 58,8 \\
- Ataque físico sin uso de arma & 59,8 & 70,7 \\
- Destrozo de objetos personales & 60,6 & 50 \\
Amenazas & & \\
- De ataque & 74,5 & 8,6 \\
Cuidadores & 50,3 & 3,1 \\
- Insulto de algún adulto cercano & & \\
- Ataques físicos de algún adulto cercano & 59,7 & 68,3 \\
Pares & 48,8 & 90,3 \\
- Ataque físico de otro NNA & & \\
- Insultos de un grupo de NNA & 13,1 & 34 \\
Sexuales & & \\
- Obligación a mirar las partes íntimas de otra persona & 82,7 & 6,2 \\
Indirectas & 82,3 & 52,3 \\
- Balaceras, microtráfico, peleas entre pandillas, etc. & & \\
- Discriminación por alguna característica propia & 22,1 & 1,1 \\
\hline Violencia intrafamiliar & Hermanos o hermanastros atacados físicamente por el padre/madre o su pareja \\
\hline
\end{tabular}

Fuente: Primera Encuesta Nacional de Polivictimización, 2017.

En el afán de indagar entre quienes estuvieron más expuestos a polivictimización, dónde se acentúa y qué rostro adquiere, la Tabla 4 presenta una caracterización de ambos perfiles, P92 y umbrales, de acuerdo con las variables teóricamente más relevantes que incluye la encuesta. A nivel general, observamos que la polivictimización por percentiles (P92) tiene una manifestación moderada entre variables sociodemográficas, siendo más intensa su relación con características psicológicas. Por el nivel de exigencia de P92, era de esperar que mostrara valores acotados en la muestra, y aunque los contrastes de chi cuadrado resultaron significativos en casi todas las variables incluidas, la polivictimización entre NNA alcanzó mayoritariamente cifras cercanas al $10 \%$ de prevalencia, sea por género, etnia, índices de vulnerabilidad escolar (IVE), dependencia del colegio, edad o nivel educativo.

Las excepciones al comportamiento descrito son las variables que miden el estado emocional (Escala de Rosenberg), anímico (Escala de Birleson) y presencia de NEE. Los más expuestos a la condición de polivictimización serían quienes alcanzan bajos puntajes en la Escala de Autoestima de Rosenberg, donde 1 de cada 5 (22\%) de ellos/as reportó que durante el último año vivió al menos 11 victimizaciones (punto de corte de P92). Prevalencia con ratios de 2,7 y 5,9 respecto a la evidenciada entre quienes alcanzan autoestima moderada y adecuada. Al interior de este subgrupo, encontramos exiguos niveles de autoconcepto y autoimagen, predominando sentimientos como sentirse inútil a veces $(92,1 \%)$, no ser bueno en absoluto $(90,0 \%)$ y percibirse como un fracaso $(86,0 \%)$, todo lo cual incrementa su receptividad, especialmente a violencia verbal como insultos o amenazas. 
TABLA 4

Caracterización de la polivictimización durante el último año según rango percentil y umbrales (\% y $\left.\mathrm{X}^{2}\right)$

\begin{tabular}{|c|c|c|c|}
\hline & Rango percentil & $\begin{array}{l}\text { Umbra } \\
\text { inte }\end{array}$ & $\begin{array}{l}\text { gún } \\
\text { d }\end{array}$ \\
\hline & P92 & Mediana & Alta \\
\hline Sexo & {$\left[X^{2}=9,91 p=, 002\right]$} & {$\left[X^{2}=33\right.$} & 000] \\
\hline - Hombre & 8,4 & 22,4 & 12,7 \\
\hline - Mujer & 9,8 & 23,7 & 14,3 \\
\hline Etnia & {$\left[X^{2}=4,54 p=, 033\right]$} & {$\left[X^{2}=3\right.$,} & 333] \\
\hline - Pertenece & 10,2 & 22,6 & 14,7 \\
\hline - No pertenece & 8,9 & 23,2 & 13,4 \\
\hline IVE & {$\left[X^{2}=8,07 p=0,018\right]$} & {$\left[X^{2}=28\right.$} & ;000] \\
\hline - Alto & 8,2 & 19,6 & 12 \\
\hline - Medio & 9,4 & 24,6 & 13,8 \\
\hline - Bajo & 9,6 & 24,8 & 14,7 \\
\hline Dependencia & {$\left[X^{2}=3,69 p=, 158\right]$} & {$\left[X^{2}=128\right.$} & $000]$ \\
\hline - Municipal & 8,9 & 21,2 & 12,9 \\
\hline - Particular subvencionado & 9,4 & 24,3 & 14,2 \\
\hline - Privada & 7,4 & 26,3 & 11,4 \\
\hline Edad & {$\left[X^{2}=19,53 p=, 000\right]$} & {$\left[X^{2}=149\right.$} & $000]$ \\
\hline - 12 a 13 años & 9 & 20,9 & 13,1 \\
\hline - $\quad 14$ a 15 años & 10,1 & 23,4 & 15 \\
\hline - 16 y más años & 7,9 & 24,6 & 12,3 \\
\hline NEE & {$\left[X^{2}=194,29 p=, 000\right]$} & {$\left[X^{2}=22\right.$} & $000]$ \\
\hline - Presenta & 16,9 & 25,6 & 22,3 \\
\hline - No presenta & 8 & 22,7 & 12,3 \\
\hline Nivel educativo & {$\left[X^{2}=3,41 p=, 065\right]$} & {$\left[X^{2}=192\right.$} & $000]$ \\
\hline - Básico & 9,5 & 20,9 & 13,8 \\
\hline - Secundaria & 8,7 & 24,7 & 13,4 \\
\hline Autoestima Rosenberg & {$\left[X^{2}=481,00 p=, 000\right]$} & {$\left[X^{2}=826\right.$} & $, 000]$ \\
\hline - Adecuada & 3,7 & 19,1 & 6,2 \\
\hline - Moderada & 8,2 & 23,1 & 12,4 \\
\hline - Baja & 22 & 29,3 & 30,6 \\
\hline Depresión de Birleson & {$\left[X^{2}=775,29 p=, 000\right]$} & {$\left[X^{2}=128\right.$} & $=, 000]$ \\
\hline - Ninguna o Baja & 5,1 & 21 & 8,6 \\
\hline - Moderada o alta & 18,4 & 28,5 & 25,4 \\
\hline
\end{tabular}

Nota: NEE = Necesidad Educativa Especial;

Fuente: Primera Encuesta Nacional de Polivictimización, 2017.

La Escala de Depresión de Birleson también resulta útil para identificar exposición a victimización. El $18,4 \%$ de quienes fueron clasificados con tendencia moderada o alta hacia la depresión, lo fueron también como polivíctimas por P92. Este subgrupo presenta niveles preocupantes de aislamiento o incomprensión, declarando sentirse muy solos $(95,0 \%)$, con ganas de llorar (94,5\%) y ganas de escapar (92,8\%). Este estado anímico que agudiza la vulnerabilidad y obstruye la búsqueda de soluciones frente a la violencia, particularmente la de tipo simbólica con sus prácticas de enajenación social. Resultados como este, sumados a lo recién mencionado sobre autoestima, expanden el campo de análisis sobre victimización, haciendo de los componentes afectivo y anímico dimensiones clave dentro de las estrategias de abordaje a la temática. 
Por su parte, la caracterización de polivictimización por umbrales exhibió mayores matices y acumulaciones que P92. Por su construcción, la mediana intensidad de polivictimización es la que más prevalencia alcanza entre NNA, con cifras superiores al $20 \%$ en la mayoría de variables incluidas en la Tabla 4 . En concreto, quienes más estuvieron expuestos a vivir entre 4 y 5 victimizaciones durante el último año son NNA de baja autoestima (29,3\%), moderada o alta depresión (28,5\%), que asisten a colegios particulares pagados $(26,3 \%)$ y que presentan alguna NEE $(25,6 \%)$. Cabe destacar que el resto de características igualmente cuenta con porcentajes preocupantes, lo que deja de manifiesto la importancia de contrastar entre criterios de clasificación para estudiar polivictimización entre NNA.

Al concentrarnos en la columna de alta intensidad de polivictimización, los valores decaen bruscamente para la mayoría de variables independientes, quedando por debajo de los recién reportados y sobre los descritos en P92. Eso sí, llama la atención las cifras de polivictimización intensa (Alta) entre quienes declararon bajos niveles de autoestima (30,6\%), moderada o alta depresión (25,4\%) y contar con alguna NEE (22,3\%), ya que, junto con ser elevadas, consistentemente destacan entre el grupo de variables, configurándose como atributos prácticamente indisociables a la condición de polivíctima. Esto sería un refuerzo a las perspectivas tradicionales de análisis al maltrato escolar, donde se plantea que el o los victimarios acosan especialmente a quienes consideran tímidos, solitarios o distintos por alguna condición especial (Galán, 2018).

En último lugar, y tratando de exponer el peso específico que un conjunto de características teóricamente relevantes tiene sobre la condición de polivictimización, se han realizado dos regresiones logísticas binomiales, una para P92 y otra para umbrales (Tabla 5). Más que buscar establecer un modelo con finalidad predictiva, el interés principal fue exponer el efecto incidental que cada regresor tiene sobre la manifestación de polivictimización, controlando los efectos de los otros predictores.

TABLA 5

Modelos de regresión binomial según criterios de polivictimización

\begin{tabular}{|c|c|c|}
\hline & $\begin{array}{l}\text { (M1) P92 } \\
\operatorname{Exp(B)}\end{array}$ & $\begin{array}{c}\text { (M2) Umbrales } \\
\operatorname{Exp}(B)\end{array}$ \\
\hline \multicolumn{3}{|l|}{ Sexo } \\
\hline - Mujer & $0,772^{* *}$ & $0,860^{* *}$ \\
\hline - Hombre & REF & REF \\
\hline \multicolumn{3}{|l|}{ IVE } \\
\hline - Bajo & REF & REF \\
\hline - Medio & 0,918 & $0,902^{*}$ \\
\hline - Alto & $0,790^{* *}$ & $0,667^{* *}$ \\
\hline \multicolumn{3}{|l|}{ NEE } \\
\hline - Presenta & $1,842^{* *}$ & $1,423^{* *}$ \\
\hline - No presenta & REF & REF \\
\hline \multicolumn{3}{|l|}{ Nivel educativo } \\
\hline - Básico & REF & REF \\
\hline - Medio & $0,865^{*}$ & $1,126^{* *}$ \\
\hline \multicolumn{3}{|l|}{ Autoestima Rosenberg } \\
\hline - Baja & $2,962^{* *}$ & $2,287^{* *}$ \\
\hline - Media & $1,680^{* *}$ & $1,357^{* *}$ \\
\hline - Adecuada & REF & REF \\
\hline \multicolumn{3}{|l|}{ Depresión de Birleson } \\
\hline - Baja & REF & REF \\
\hline - Moderada o alta & $3,302^{* *}$ & $2,338^{* *}$ \\
\hline Constante & 0,042 & 0,362 \\
\hline \multicolumn{3}{|l|}{ Índices de ajuste } \\
\hline R cuadrado de Nagelkerke & 0,111 & 0,093 \\
\hline Clasificación global & 91 & 66,2 \\
\hline
\end{tabular}

Nota: ${ }^{* *}=$ valor $\mathrm{p}<, 01 ;{ }^{*}=$ valor $\mathrm{p}<, 05$.

Fuente: Primera Encuesta Nacional de Polivictimización, 2017. 
Al analizar el modelo de polivictimización por percentiles (M1), encontramos que ser mujer, asistir a colegios con altos niveles de vulnerabilidad y encontrarse en enseñanza media, disminuyen la exposición a violencia y victimización respecto a quienes no poseen estas características. Por su parte, presentar depresión, bajos niveles de autoestima y alguna NEE, no solo incrementan el riesgo a experimentar victimización, sino que se constituyeron en las variables de mayor relevancia. Todos los factores analizados, a excepción del sexo, mantuvieron el comportamiento ya observado en el contexto bivariante. En términos específicos, presentar depresión según la Escala de Birleson incrementa significativamente la posibilidad de constituirse en polivíctima $(\operatorname{Exp}(B)=3,302 ; \mathrm{p}<, 01)$; en igual sentido, pero con menor incidencia, lo son tener baja autoestima según Escala de Rosenberg $(\operatorname{Exp}(B)=2,962 ; p<, 01)$ y declarar alguna $\operatorname{NEE}(\operatorname{Exp}(B)=1,842 ; p<, 01)$.

En el caso del modelo de regresión por umbrales (M2), se dicotomizó el perfil entre quienes presentaban baja o nula victimización ( 3 o menos) y aquellos con mediana o alta polivictimización (4 y más). Este modelo resulta similar al M1, de hecho, se mantienen tanto la orientación como la jerarquía de las variables independientes, con excepción de cursar enseñanza media, que ahora resulta un factor de exposición a polivictimización $(\operatorname{Exp}(B)=1,126 ; p<, 01)$. Las probabilidades vinculadas con depresión, autoestima y NEE disminuyen ligeramente, pero siguen siendo factores que incrementan notoriamente la exposición a violencia y victimización.

La interpretación general de estos datos apoya el comportamiento espiral de la polivictimización, donde la exposición a violencia se vincula directamente con la vulnerabilidad de los NNA, especialmente en la escuela. Encontramos distintos tipos de violencia, los cuales interactúan con las características psicológicas o demográficas de los y las estudiantes, por lo que abordajes tradicionales como el de maltrato escolar vinculado con agresiones físicas resulta insuficiente para captar la multidimensionalidad del problema. Esto involucra replantearse los roles que tienen los actores que forman parte de la comunidad educativa, entre los que destacan los pares como ejecutores y testigos de victimización, mientras que los cuidadores son potenciales fuentes de riesgo. En suma, la EPV reportó una situación preocupante y, al parecer, fuera de las manos para cualquier gestión educativa, aunque, como veremos a continuación, deben hacerse algunas puntualizaciones y establecer matices que moderan el alarmismo.

Los diferentes tipos de victimización que mide la EPV a través de sus dimensiones e indicadores proyectan la situación de vulnerabilidad y tipos de violencia más recurrentes entre NNA en diferentes contextos, además de incluir características individuales y estructurales para su identificación.

La figura 1 es una matriz de relaciones entre familias de conceptos vinculados a lo encontrado en los datos, según criterios de clasificación como factores de espiral, formas de violencia, roles en el maltrato escolar y tipos de actor. Lo primero que debemos destacar es que entre violencia, vulnerabilidad y victimización existe un vínculo de apoyo recíproco, siendo en el campo social un ejemplo de lo que en psicología se llama comorbilidad, es decir, síntomas que se presentan simultáneamente en las personas sin poder determinar concretamente cuál fue primero y cuál después (Flores, 2009). Si a esto añadimos el hallazgo de que tanto en el hogar, el barrio y la escuela se viven hechos de victimización, entonces el prisma de observación se convierte en un caleidoscopio donde se vuelve complicado determinar cuál es la fuente primaria y cuáles los refuerzos de la violencia o vulnerabilidad.

Aún más, a esta comorbilidad debemos añadir las características afectivas y anímicas que cada NNA forja en respuesta a sus condiciones de vida. Según las cifras de la EPV, la victimización en la escuela, lugar privilegiado de análisis, es más recurrente entre quienes se encuentran en condiciones de baja autoestima y tendencias depresivas, seguido de la presencia de Necesidades Educativas Especiales. Sin poder establecer qué fue primero, si la agresión o el escaso valor autopercibido, de lo que no hay dudas es que uno retroalimenta al otro y crecen a medida que se repite la victimización, la exposición a violencia y la vulnerabilidad (Carbajal, 2010). 
Como si esto no fuera ya preocupante, debemos mencionar la situación de los NNA polivíctimas, perfil de interés para este trabajo, y entre quienes la espiral de riesgo descrita amenaza con volverse parte de su cotidianidad. Este grupo no tan solo reporta haber estado expuesto a situaciones que pusieron en riesgo su integridad física, sino también a violencia verbal y simbólica, donde los insultos y las amenazas parecen constituyentes de la socialización que llevan a cabo en la escuela. A nivel individual, la vivencia sistemática de violencia bajo sus distintas formas supone hacer del temor una política de vida, además que fortalece la desensibilización de los actores involucrados, tanto victimario y testigos, como entre las víctimas. Lo que declaran las polivíctimas dibuja un escenario árido y complejo de relación con otros, especialmente si consideramos que las experiencias de violencia reiterada afectan profundamente la forma cómo comprendemos que funcionan las relaciones sociales (Varela, 2012).

Figura 1. Malla de conceptos vinculados a la polivictimización en la escuela

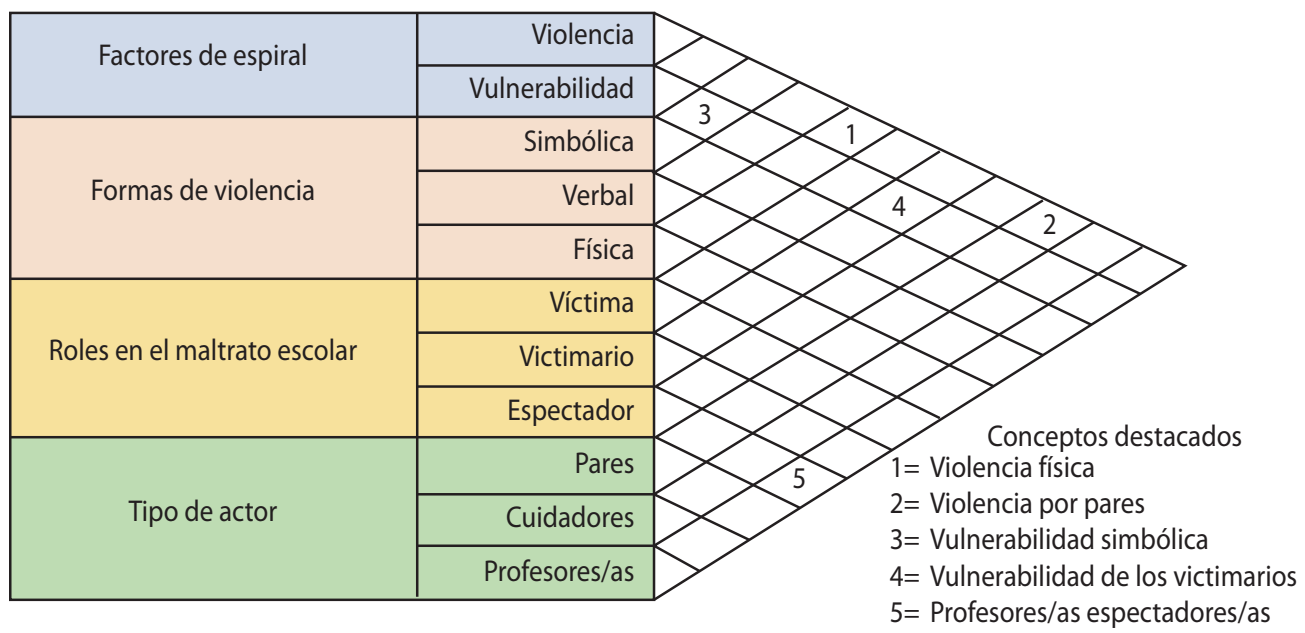

Fuente: Elaboración propia.

Un tercer elemento que añadir es el efecto potenciador que tienen sobre las problemáticas mencionadas la pobreza y la desigualdad, condiciones que en Chile tienen una marcada distribución territorial y de clase (Agostini, Brown y Góngora, 2008; Larraín, 2008). Y no nos referimos tan solo a la relación entre tipo de educación a la cual se adscribe y disponibilidad de recursos socioeducativos, aunque es válido comenzar por aquí. En varias ocasiones se ha descrito que violencia, maltrato y algunos actos de delincuencia, se encuentran localizados en determinados tipos de establecimiento, que no son propios al sistema escolar sino que responden a determinadas características de la unidad educativa (Tijmes, 2012; Castañeda, 2018). Sin embargo, dado el contexto de profunda segmentación, estas unidades suelen ser las que atienden mayoritariamente a población de bajos ingresos, se ubican en sectores barriales o periurbanos y alcanzan resultados insuficientes en las mediciones de calidad educativa (López y otros, 2011).

En tal sentido, los resultados de la EPV son reveladores, ya que mostraron cómo la victimización y la violencia son hechos ubicuos, más correlacionados con atributos individuales que con las características del lugar en que los NNA viven y estudian. Ambas problemáticas serían connaturales a la interacción que establecen los y las estudiantes en cualquier espacio educativo, con cierta independencia de la tipología del establecimiento o de las características sociofamiliares y territoriales de las víctimas o los victimarios. Este hallazgo debate contra la focalización que los estudios sobre victimización y delincuencia juvenil han tenido sobre los colegios pobres y vulnerables que se ubican en sectores marginales, planteando que cualquier relación social entre NNA involucra potencialmente violencia en alguna de las múltiples formas que incluye la EPV. Eso sí, no podemos obviar que el sustento de esta postura refiere a la sensibilidad métrica de un instrumento que no discrimina con base a la intensidad del item que tipifica la 
violencia, asignando la misma puntuación a un insulto que a un ataque físico, o aún más grave, a una vulneración sexual. De igual modo, no diferencia entre el atestiguamiento y la vivencia de victimización, lo que provoca una sobreestimación del fenómeno.

Ahora, respecto a los tipos de violencia, los indicadores de ataques y golpes alcanzaron elevadas cifras entre NNA de forma reiterada en cada una de las tablas presentadas. En especial, los pares serían una fuente relevante de producción de violencia, lo cual se urde con las declaraciones generales de victimización indirecta y amenazas, lo que hace suponer que una cantidad importante de NNA vive y ejerce violencia en la escuela, tal y como observan o reciben esta en casa o sus alrededores. Debido a la proximidad que tienen los y las estudiantes en la escuela, debemos asumir también que el conflicto es inevitable, tal y como lo es en los lugares de trabajo o aglomeración pública entre adultos. Sin embargo, hay consenso en que estas discusiones o peleas no pueden sobrepasar los límites del diálogo y la resolución pacífica, especialmente porque es en la escuela donde se aprende a convivir y relacionarse con los otros, cómo llegar a acuerdos y con qué medios resolver los inconvenientes (Del Rey, Ortega y Feria, 2009).

Por tanto, es imprescindible fomentar entre los y las estudiantes un clima de convivencia escolar y áulico que cumpla con criterios de seguridad, respeto y organización, tal y como reconoce el Ministerio de Educación que debería funcionar cada salón de clases (Ministerio de Educación, 2014). La violencia e inseguridad interna o externa del colegio impactan negativamente sobre los procesos de aprendizaje y desarrollo emocional, obstaculizando el involucramiento de los NNA en su vida escolar y social. Por lo demás, la presencia de estas problemáticas en la escuela dificulta la labor educativa, ya que obliga a redireccionar recursos, particularmente tiempo y atención pedagógica, desde la enseñanza hacia la disciplina o mediación en problemas sociofamiliares (Trucco y Inostroza, 2017; Castañeda, 2018).

Por otra parte, como en cualquier discusión sobre victimización y maltrato escolar, no podríamos dejar de lado la problematización de la icónica figura del victimario o bully, a quien suele situarse en el centro de comprensión de estas temáticas. El campo actual de investigación y gestión educativa comprende al maltratador como un actor rodeado por circunstancias complejas que, de forma directa o simbólica, refuerzan su comportamiento agresivo, además que recompensan su labor con respeto y seguridad de que otro no le someterá (Paulín, 2015). Aunque con la EPV no se puede calar profundo en los rasgos de quien maltrata, sí podemos argumentar que, debido a los niveles generales de victimización y los resultados de las escalas de Autoestima y Depresión, muy probablemente los NNA que ejercen y reciben victimización comparten un perfil de riesgo y exposición a violencia simliar. Tal y como exponen García y Ascensio (2015), el maltrato escolar es un problema dinámico donde los papeles se pueden intercambiar constantemente, de modo que quien se considera victimario puede ser a la vez víctima de otros NNA y estar atestiguando o instigando actos de victimización.

Considerando que la construcción del índice de polivictimización se sitúa en lo ocurrido los últimos doce meses, los delitos comunes y la victimización indirecta parecen formar parte de la cotidianidad entre los NNA que comparten este perfil. Supuesto que tendría su correlato en los bajos niveles de autoestima y tendencias depresivas que declararon las polivíctimas, fenómenos que, además de requerir largo tiempo para acentuarse, tienen a la base de su emergencia y arraigamiento la vivencia de situaciones que atentan contra el cuerpo, la identidad y el involucramiento con el resto (Massenzana, 2017). No es aventurado afirmar que la exposición a violencia física entre polivíctimas impacta profundo en su comprensión sobre sí mismos, llegando a justificar tal agresividad en la propia debilidad, timidez o desajuste al grupo. Como bien expone Horna (2017), el rechazo reiterado que se expresa en golpes y ataques puede llegar a provocar una renuncia a cualquier defensa, un convencimiento del poco valor que se tendría para los otros.

Las consecuencias de un clima escolar agresivo son variadas y van incluso más allá de las víctimas y su autoestima, afectando los procesos de aprendizaje y la puesta en ejercicio de las habilidades sociales del grupo (Cordano, 2017). Además, el sentido de pertenencia y la eficacia que pueden tener figuras como la del docente o los profesionales asistentes de la educación se reduce a medida que se visibilizan en 
el ambiente escolar hechos de victimización. Con todo, es primordial erradicar los actos de maltrato en el espacio educativo, aunque no resulta menos cierto que la sociedad en general cada vez más parece seducida por la producción y distribución de violencia en sus distintos tipos (Giró, 2006).

En síntesis, la polivictimización está influenciada por una serie de factores en distintos niveles. Están aquellas características de los NNA que incrementan su exposición, como la baja autoestima, la presencia de Necesidades Educativas Especiales y las tendencias depresivas; algunos atributos de los espacios donde se desarrollan, como son la escuela, el barrio y el hogar, donde pueden estar fomentando una desensibilización o producción de violencia; y, por último, el refuerzo que hacen los otros a las prácticas de victimización.

\section{SÍNTESIS Y REFLEXIONES FINALES}

Como una síntesis de lo discutido en las secciones previas, hay dos hallazgos que destacar. El primero es que la victimización reportada por estudiantes durante el último año alcanza valores preocupantes, lo cual permite afirmar que es un hecho extendido por lo largo y ancho del sistema escolar en las distintas formas que la EPV considera.

Cabe destacar que, como este trabajo solo considera aquellos indicadores o eventos que podían ocurrir en la escuela, la inclusión de todos los ítems al análisis podría incrementar aún más las cifras generales de victimización, con el añadido que daría a los perfiles de polivictimización otros lugares de atención respecto a su vulnerabilidad y exposición a violencia.

Así mismo, la presencia de polivíctimas, fueran definidas según percentil de distribución (P92) o por declarar una cantidad mínima de ellos (umbrales), es transversal entre grupos etarios, niveles educativos y tipologías de colegio, lo cual acentúa el dinamismo de la manifestación y vivencia de victimización entre estudiantes.

Sin embargo, aunque podemos aseverar que estas problemáticas no tienen sede fija y que lamentablemente aquejan a la mayoría de quienes respondieron la encuesta, ello no implica que las formas e intensidad de la violencia estén igualmente distribuidas.

Al respecto, lo más urgente de atender sería la prevalencia con que son declarados a nivel general los delitos comunes, la victimización indirecta y la ejercida por pares, todo lo cual implica un perjuicio físico, psciológico, social y afectivo para los y las estudiantes.

La gestión educativa tiene aquí una tarea difícil de cumplir, puesto que no tan solo debe preocuparse por asegurar el bienestar físico de los NNA en el aula y el colegio, sino que debido a las relaciones dinámicas entre los distintos tipos de violencia, el frenar los ataques o golpes entre pares no asegura que las víctimas no estén expuestas a violencia verbal o simbólica en los sitios donde el profesor/a o inspector/a no puede llegar.

La violencia tiene una serie de apoyos recíprocos, donde a la violencia física le secundan o preceden actos de maltrato verbal o aislamiento, además que la escuela puede ser uno más de los espacios donde las víctimas o los victimarios ejercen o reciben violencia.

Esta multidimensionalidad de la victimización tiene al menos tres niveles: el más obvio es el que afecta a las víctimas, quienes pueden vivir al mismo tiempo más de un tipo de maltrato físico, verbal, psicológico, simbólico o sexual; el segundo correspondería a los contextos o espacios donde se desenvuelven, pudiendo ser víctimas en casa, el barrio o la escuela; por último, estarían los perpetradores, quienes pueden ser los cuidadores, amigos, pares, cercanos, familiares, etc. Todo esto hace de la polivictimización 
una problemática difícil de atender satisfactoriamente, algo más allá de los límites de injerencia de la escuela, pero que, sin dudas, le exige acciones.

Esto nos lleva al segundo punto a destacar: el rol de los pares en el ejercicio de la violencia. Las acciones que llevan a cabo los compañeros/as de escuela y clase puede ser algo en lo que intervenir activamente para frenar los índices de victimización reportada entre NNA.

Los pares son activos productores de agresiones, ataques y amenazas, lo cual, además de afectar directamente a las víctimas convierte al espacio escolar en un sitio inseguro y displacentero para los y las estudiantes. A juzgar por los valores generales de victimización, podemos afirmar también que tanto victimarios como víctimas están insertos en contextos de violencia y vulnerablidad donde hay pautas de agresividad que se reproducen continuamente.

Para finalizar, se debe reiterar que tanto victimización como polivictimización son problemáticas cuya raigambre excede a la escuela; sin embargo, este es un lugar clave desde el cual enfrentarlas.

Tal vez los resultados y discusiones aquí expuestas proyecten un escenario árido y hostil que daría pie a pensar la escuela como un espacio de riesgo para los y las estudiantes, lo cual no sería exagerado para quienes caen dentro del perfil de polivictimización y que, además de en la escuela, deben afrontar vulneraciones en su barrio o el hogar, pero a nivel general sostenemos el carácter reflexivo y práctico de lo dicho.

En otras palabras, los resultados expuestos deben ser considerados a la luz de un instrumento autoreportado que contiene numerosos indicadores y cuya métrica es altamente sensible, lo que provoca que las polivíctimas no respondan necesariamente a los episodios de mayor gravedad.

Por último, la utilidad de este estudio reside en las ventanas de oportunidad que abre a la gestión escolar y comunitaria, proveyendo información clara y representantiva del sistema, complementando estudios de caso o comparativos que entregan diagnósticos locales que, dada su especificidad, tienen un alcance limitado. Con ello se perfila una línea de investigación y de intervención de profundo interés para futuros estudios de violencia y victimización en el campo educativo.

\section{REFERENCIAS}

Agostini, C., Brown, P., \& Góngora, D. (2008). Spatial distribution of poverty in Chile. Estudios de economía, 35(1), 79-110. https://estudiosdeeconomia.uchile.cl/index.php/EDE/article/view/40238/43432

Cañas-Pardo, E. (2017). Acoso escolar: características, factores de riesgo y consecuencias. Revista Doctorado UMH, 3(1), 7. DOI: 10.21134 / doctumh.v3i1.1371

Carbajal, P. (2010). La convivencia como alternativa a la violencia escolar: experiencias exitosas desde Latinoamérica. Guanajuato: Conferencia en el VII Encuentro Participación de la Mujer en la Ciencia. Centro de Investigaciones en Óptica (memoria en disco compacto).

CASTAÑEDA, G. (2018). Maltrato escolar, práctica pedagógica y lenguaje. Enunciación, 23(2), 162-179. DOI: https://doi.org/10.14483/22486798.13187

ChAN, K., YAN, E., \& Brownridge, D. (2013). Associating child sexual abuse with child victimization in China. The Journal of pediatrics, 162(5), 1028-1034.

DOI: 10.1016 / j.jpeds.2012.10.054

ChenG, P.-X., CAO, F.-L., LIU, J.-J., \& CHEN, Q.-Q. (2010). Reliability and validity of Chinese self-report version of Juvenile Victimization Questionnaire in middle school students. Chinese Journal of Clinical Psychology, 18(4), 460-462. 
CoRdAno, M. (08 de Mayo de 2017). El sentido de pertenencia al colegio es un factor clave en el bienestar de los alumnos.

http://www.economiaynegocios.cl/noticias/noticias.asp?id=358589

Del Rey, R., Ortega, R., \& Feria, l. (2009). Convivencia escolar: fortaleza de la comunidad educativa y protección ante la conflictividad escolar. Revista interuniversitaria de formación del profesorado(66), 159-180. https://core.ac.uk/download/pdf/51383856.pdf

Echeverri, M., y Vargas-González, J. (2018). Actores del acoso escolar. Revista Médica de Risaralda, 24(1), 60-66.

http://www.scielo.org.co/scielo.php?script=sci_arttext\&pid=S0122-06672018000100011\&lng=e n\&tlng=es.

Finkelhor, D., Hamby, S., Ormrod, R., \& Turner, H. (2005). The Juvenile Victimization Questionnaire: Reliability, validity, and national norms. Child Abuse \& Neglect, 29, 383-412. DOI: 10.1016 / j.chiabu.2004.11.001

Finkelhor, D., Shattuck, A., Turner, H., Ormrod, R., \& Hamby, S. (2011). Polyvictimization in developmental context. Journal of Child \& Adolescent Trauma, 4(4), 291-300. https://doi.org/10.1080/19361521.201 1.610432

FLORES, J. (2009). Características de comorbilidad en los diferentes subtipos de trastorno por déficit de atención con hiperactividad. Psicothema, 21(4), 592-597.

http://www.psicothema.com/PDF/3676.pdf

GALÁN, J. (2018). Exposición a la violencia en adolescentes: desensibilización, legitimación y naturalización. Diversitas, 14(1), 55-67.

http://www.scielo.org.co/pdf/dpp/v14n1/1794-9998-dpp-14-01-00055.pdf

Garaigordobil, M., \& OñederRa, J. (2008). Estudios epidemiológicos sobre la incidencia del acoso escolar e implicaciones educativas. Información Psicológica, 94, 14-35.

http://www.informaciopsicologica.info/OJSmottif/index.php/leonardo/article/viewFile/236/190

GARCiA, M., \& AsCENSIO, C. (2015). Bullying y violencia escolar: diferencias, similitudes, actores, consecuencias y origen. Revista Intercontinental de Psicología y Educación, 17(2), 9-38.

https://www.redalyc.org/pdf/802/80247939002.pdf

GIRó, X. (2006). Los medios y la cobertura de la violencia. En PNUD (Ed.), IV Ciclo de Conferencias - Aportes para la convivencia y la seguridad ciudadana (Vol. II, págs. 187-204). http://www.pnud.org.sv/2007/component/option,com_docman/task,cat_view/gid,19/dir,DESC/ order,date/Itemid,56/limit,10/limitstart,10/

Guerra, C., Inostroza, R., Villegas, J., Villalobos, L. y Pinto-Cortez, C. (2017). Polivictimización y sintomatología postraumática: el rol del apoyo social y la autoeficacia. Revista de psicología (Santiago), 26(2), 66-75. https://dx.doi.org/10.5354/0719-0581.2017.47951

HORNA, V. (2017). Un estudio cualitativo sobre convivencia escolar: El Bullying desde la perspectiva de las Víctimas. ConCiencia EPG , 2(2), 46-56.

DOI 10.32654/CONCIENCIAEPG.2-2.4

Kirchner, T., Soler, L., \& Paretilla, C. (2013). Spanish/catalán versión of the Juvenile Victimization Questionnaire (JVQ): Psychometric properties. Anuario de psicología/The UB Journal of psychology, 171-188.

Kretschmar, J. M., Tossone, K., Butcher, F., \& Flannery, D. J. (2017). Patterns of poly-victimization in a sample of at-risk youth. Journal of Child \& Adolescent Trauma, 10(4), 363-375.

Larraín, F. (2008). Cuatro millones de pobres en Chile: Actualizando la línea de la pobreza. Estudios Públicos(109), 101-148.

https://www.cepchile.cl/cep/estudios-publicos/n-91-a-la-120/estudios-publicos-n-109-2008/

cuatro-millones-de-pobres-en-chile-actualizando-la-linea-de-pobreza 
López, V., Carrasco, C., Morales, M., Ayala, Á., López, J., \& Karmy, M. (2011). Individualizando la Violencia Escolar: Análisis de Prácticas Discursivas en una Escuela Municipal de la Región de Valparaíso. Psykhe, 20(2), 7-23. DOI:10.4067/S0718-22282011000200002

LosAdA, A. V., \& Porto, M. (2019). Familia y abuso infantil. Revista Neuronum, 5(2), 7-32.

MASSENZANA, F. (2017). Autoconcepto y autoestima: ¿sinónimos o constructos complementarios? \{PSOCIAL\}, 3(1), 39-52. https://publicaciones.sociales.uba.ar/index.php/psicologiasocial/article/viewFile/2336/1984

Ministerio de Educación de Chile. (2014). Otros indicadores de calidad educativa. Santiago de Chile: Unidad de Currículum y Evaluación. http://archivos.agenciaeducacion.cl/resultados-simce/fileadmin/Repositorio/2014/OIC_Resultado_ Establecimientos/OIC_IRE_2014_RBD-9004.pdf

Paulín, H. (2015). Hacia un enfoque psicosocial crítico de la violencia escolar Aportes desde un estudio con estudiantes de la ciudad de Córdoba, Argentina. Universitas Psychologica, 14(5), 1751-1762. http://www.scielo.org.co/pdf/rups/v14nspe5/v14nspe5a17.pdf

Pereda, N., y Forns, M. (2007). Prevalencia y características del abuso sexual infantil en estudiantes universitarios españoles. Child Abuse \& Neglect, 31, 417-426. http://diposit.ub.edu/dspace/bitstream/2445/34562/1/551047.pdf

Pereda, N., Gallardo-Pujol, D. \& Guilera, G. (2018). Good practices in the assessment of victimization: The Spanish adaptation of the Juvenile Victimization Questionnaire. Psychology of Violence, 8(1), 7686. Doi: $10.1037 /$ vio000007

Pinto-Cortez, C., Flores-Jara, J., Pereda, N. y Guerra, C. (2019). Victimización y polivictimización en niños, niñas y adolescents aymara y su relación con sintomatología post-traumática. INTERCIENCIA, 44(4), 229-235. Recuperado de https://www.redalyc.org/jatsRepo/339/33959119007/html/index. $\mathrm{html}$

RojAS, F., y SieRRA, C. (2013). Manifestaciones de violencia en la escuela primaria: elementos de perfilación de víctimas y agresores. Revista Panorama, 3(7), 98-116. DOI:10.15765/PNRM.V3I7.218 Corpus ID: 145537936

SALGADO, C. (2017). El derecho de los niños a una educación de calidad. En UNICEF, Constitución política e infancia. Una mirada desde los niños, niñas y adolescentes en Chile (págs. 291-329). Santiago de Chile: UNICEF.

https://www.unicef.org/chile/informes/constitucion-politica-e-infancia-una-mirada-desde-losderechos-de-los-ninos-ninas-y

Sterzing, P.R., Ratliff, G.A., Gartner, R.E., McGeough, B.L. \& Johnson, K.C. (2017). Social Ecological Correlates of Polyvictimization among a National Sample of Transgender, Genderqueer, and Cisgender Sexual Minority Adolescents. Child Abuse \& Neglect, 67,1-12. doi: 10.1016/j.chiabu.2017.02.017

Subijana, I. y Echeburúa, E. (2018). Los menores víctimas de abuso sexual en el proceso judicial: el control de la victimización secundaria y las garantías jurídicas de los acusados. Anuario de Psicología Jurídica, 28, 22-27.

TIJMES, C. (2012). Violencia y clima escolar en establecimientos educacionales en contextos de alta vulnerabilidad social de Santiago de Chile. Psyhke, 21(2), 105-117. https://scielo.conicyt.cl/scielo.php?script=sci_arttext\&pid=S0718-22282012000200009

Tresgallo, E. (2011). El acoso escolar y las actitudes psicológicas defensivas en el primer ciclo de Educación Primaria. Revista Española de Orientación y Psicopedagogía, 22(1), 28-37. https://www.redalyc.org/pdf/3382/338230787004.pdf

Trucco, D., y Inostroza, P. (2017). Las violencias en el espacio escolar. Santiago de Chile: Naciones Unidas. https://repositorio.cepal.org/bitstream/handle/11362/41068/4/S1700122_es.pdf 
Tur-Porcar, A., Mestre, V., Samper, P., \& Malonda, E. (2012). Crianza y agresividad de los menores: ¿es diferente la influencia del padre y de la madre? Psicothema, 24(2), 284-288. Tur- Porcar, A., Mestre, V., Samper, P., \& Malonda, E. (2012). Crianza y agresividad de los menores: ¿es diferente la influencia del padre y de la madre? Psicothema, 24(2), 284-288.

VARELA, R. (2012). Violencia, Victimización y Cyberbullying en adolescentes escolarizados/as: una perspectiva desde el Trabajo Social. Sevilla: Facultad de Ciencias Sociales, Departamento de Trabajo Social y Servicios Sociales.

http://hdl.handle.net/10433/4127

Villalobos, B., Carrasco, C., Olavarría, D., Ortiz, S., López, V., Oyarzún, D., .. Álvarez, J. P. (2016). Victimización de pares y satisfacción con la vida: la influencia del apoyo de profesores y compañeros de la escuela. Psykhe, 25(2), 1-16.

http://www.psykhe.cl/index.php/psykhe/article/view/861 